\title{
A Novel Method for Improving Water Injectivity in Tight Sandstone Reservoirs
}

\author{
Mohamad Yousef Alklih, ${ }^{1}$ Bisweswar Ghosh, ${ }^{1}$ and Emad Waleed Al-Shalabi ${ }^{2}$ \\ ${ }^{1}$ Petroleum Engineering Department, The Petroleum Institute, P.O. Box 2533, Abu Dhabi, UAE \\ ${ }^{2}$ Department of Petroleum \& Geosystems Engineering, The University of Texas Austin, Austin, TX 78712, USA
}

Correspondence should be addressed to Mohamad Yousef Alklih; moyalklih@pi.ac.ae

Received 15 July 2014; Accepted 23 August 2014; Published 21 September 2014

Academic Editor: Yunho Hwang

Copyright (C) 2014 Mohamad Yousef Alklih et al. This is an open access article distributed under the Creative Commons Attribution License, which permits unrestricted use, distribution, and reproduction in any medium, provided the original work is properly cited.

\begin{abstract}
Applicability of electrokinetic effect in improving water injectivity in tight sandstone is studied. DC potential and injection rate are varied for optimization and determination of their individual impact on clay discharge and movement. The liberated clays were characterized through size exclusion microfiltration and ICP-MS analysis. Real time temperature and $\mathrm{pH}$ monitoring were also informative. Results showed that severalfold (up to 152\%) apparent increase of core permeability could be achieved. Some of the experiments were more efficient in terms of dislodgement of clays and enhanced stimulation which is supported by produced brines analysis with higher concentration of clay element. The results also showed larger quantity of clays in the produced brine in the initial periods of water injection followed by stabilization of differential pressure and electrical current, implying that the stimulation effect stops when the higher voltage gradient and flow rates are no more able to dislodge remaining clays. Additionally, fluid temperature measurement showed an increasing trend with the injection time and direct proportionality with the applied voltage. The basic theory behind this stimulation effect is predicted to be the colloidal movement of pore lining clays that results in widening of pore throats and/or opening new flow paths.
\end{abstract}

\section{Introduction}

In general, the three phases of petroleum recovery processes are primary, secondary, and tertiary oil recovery. The primary recovery phase is mainly driven by the natural energy present in the reservoir due to dissolved solution gas pressure, pressure from the overlain gas cap, or the pressure from an active aquifer below the oil zone. In most cases, the natural driving mechanism is a relatively inefficient process and results in a low overall oil recovery [1]. The lack of sufficient and consistent natural driving energy is compensated by supplementing with injection of water or gas (or a combination of both) which is the initiation of the secondary recovery phase. Due to several technoeconomic factors, the most widespread secondary oil recovery process responsible for most of world's oil production is the waterflood recovery [2]. Nevertheless, water injection may be associated with numerous hurdles related to fluid incompatibility, reservoir heterogeneity, early breakthrough through thief zones, permeability damage due to suspended particles, and clay swelling.
Poor water injectivity is one of the problems often encountered especially in tight formations [3]. This problem may further aggravate, when swelling type clays are present due to which pore throat blockage can occur [4]. This situation is often faced in clastic shaly sandstones. The swelling and release of clay particles from pore walls and their subsequent redeposition downstream in smaller pore throats may induce severe injectivity damage $[5,6]$.

The current practice to enhance water injectivity in tight and clayey formation is centered around hydraulic fracturing and matrix acidizing. Unfortunately, environmental concerns and difficulties to achieve the desired fracture length and orientation may discourage hydraulic fracturing [7]. In most cases, matrix acidizing could be challenging to implement in clay rich sandstone formations due to the complex reactions between hydrofluoric acid (HF) and the rock minerals (silicates, feldspars, and chlorites) and the damage that may occur by secondary precipitation particularly at high temperatures $[8,9]$. 


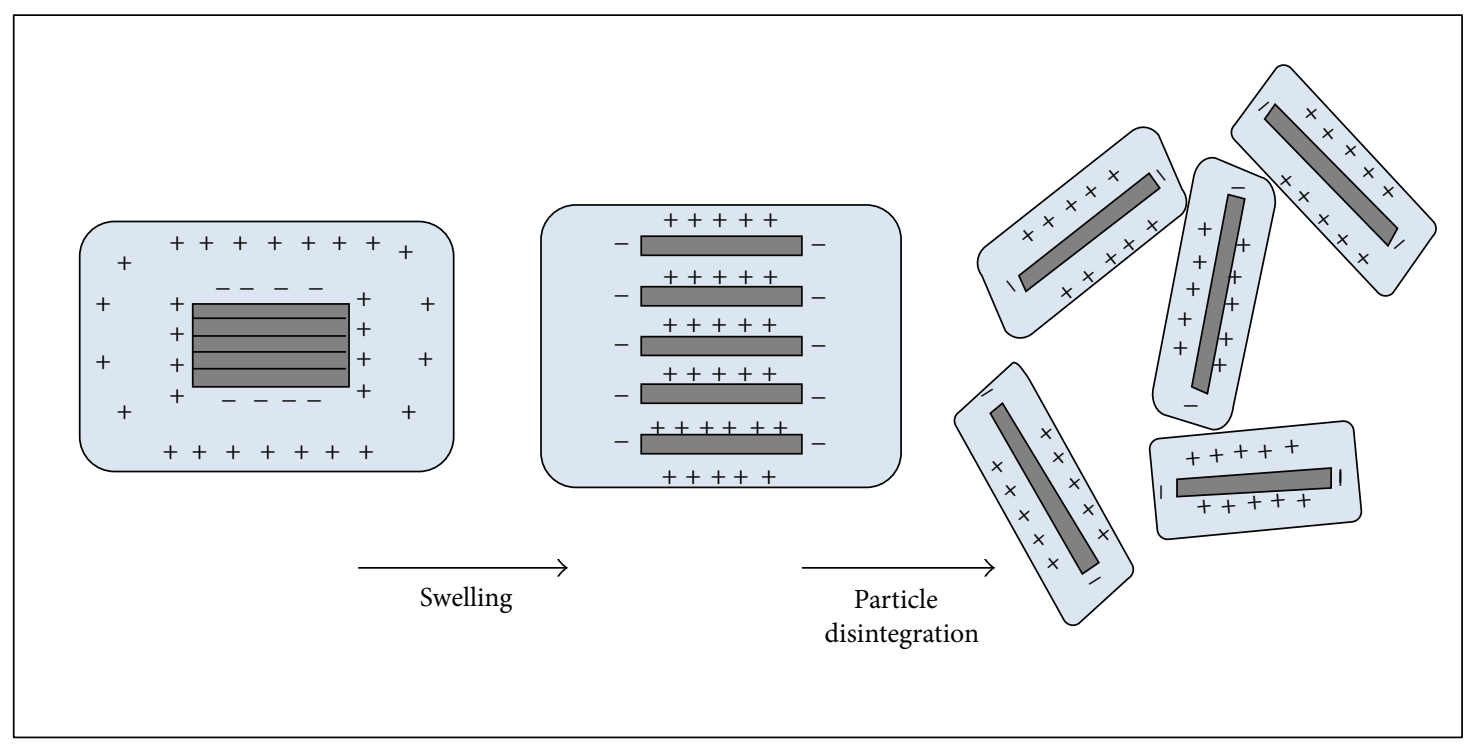

FIGURE 1: The mechanism of clay disintegration [19].

In view of the above, we studied an alternative stimulation technology to improve water injectivity in tight and clay rich sandstone reservoir with the application of electrokinetic potential. The application of electrokinetic phenomena in improving oil recovery from heavy oil field was first proposed and experimentally verified in 1960s by Chilingar and his associates [10-12]. In principle, it involves passage of direct current through an oil bearing formation resulting in complex electrokinetic transport phenomena involving electromigration, electrophoresis, electroosmosis, electrochemical reactions, and Joule heating. All of these mechanisms contribute to improvement of fluid flow in porous media [12].

Electromigration is basically the gradual movement of ions that occurs between the anode and cathode. Electrophoresis induces the separation and movement of the negatively charged clay particles in the form of colloids or surface charged micelles. Electroosmosis is the movement of an ionic solution (such as brine) in a porous media by the application of electrical potential [13]. The electroosmosis flow is caused by Coulomb's force that is present in a mobile and electrically charged solution. The electrochemically enhanced reactions occur due to the interaction between the fluid host material (matrix) and the pore fluid. These reactions are affected by the changes in the electrical potential, ionic strength, and $\mathrm{pH}$ of mobile fluid [14]. Due to these electrokinetic phenomena, dislodgement and migration of clay may occur as colloids of submicron size, which may directly stimulate rock permeability and increase effective porosity. The main mineralogical components of clay minerals are the phyllosilicates. Phyllosilicates are aluminum or magnesium silicates with a certain degree of isomorphous substitution of the principal cations that can give a net electrical charge to the solid. The basic components which form the phyllosilicates are silica $\left(\mathrm{SiO}_{2}\right)$ tetrahedral sheets and aluminum $(\mathrm{Al})$ or magnesium $(\mathrm{Mg})$ oxides octahedral sheets. Sheets can be stacked and arranged in different forms, forming different clay minerals. The positive charges that are distributed on the edges balance the negative charges that are distributed on the flat surfaces of the layers [15]. When water is flooded into the system, the osmotic pressure differential of the water tends to pull the negatively charged ions along the surface of the layer. Nevertheless, the electrostatic charges that are associated with the negative surface charges tend to hold against the osmotic pressure. Eventually, an electrical double layer is formed after a steady state is established between the osmotic pressure and the electrostatic pull [16]. The double layer obviously consists of two layers. One layer is fixed and composed of positive charges bonded to the negative surface charges, forming a strong linear bond, and the other layer is mobile composed of positive ions and some slackly bonded ions. Between these two layers, there exists a slipping surface which let the mobile layer move without disturbing the fixed layer [17]. This leads to migration of clay as colloidal particles $(<1 \mu \mathrm{m}$ size) upon introduction of the hydrodynamic flow (supported by electroosmotic flow) and eventually leading to pore throat enlargement and permeability enhancement. This phenomenon is depicted schematically through Figure 1. The electroosmotic flow rate is directly proportional to applied potential gradient and zeta potential, but inversely proportional to ion concentration, valence number, and viscosity of the solution [18]. Under electric potential the total flow rate (hydrodynamic and electroosmotic) through a porous media as proposed by Chilingar et al. is given in [15]

$$
q_{t}=\frac{A K \Delta P}{\mu L}+\frac{A K_{e} E}{\mu L} .
$$

The first component of the right hand side of the equation is the hydrodynamic flow or the Darcy flow equation. The second component suggests that the increase in the flow rate due to the application of electrokinetic phenomena is dependent on the zeta potential, dielectric constant, and electric field strength. 


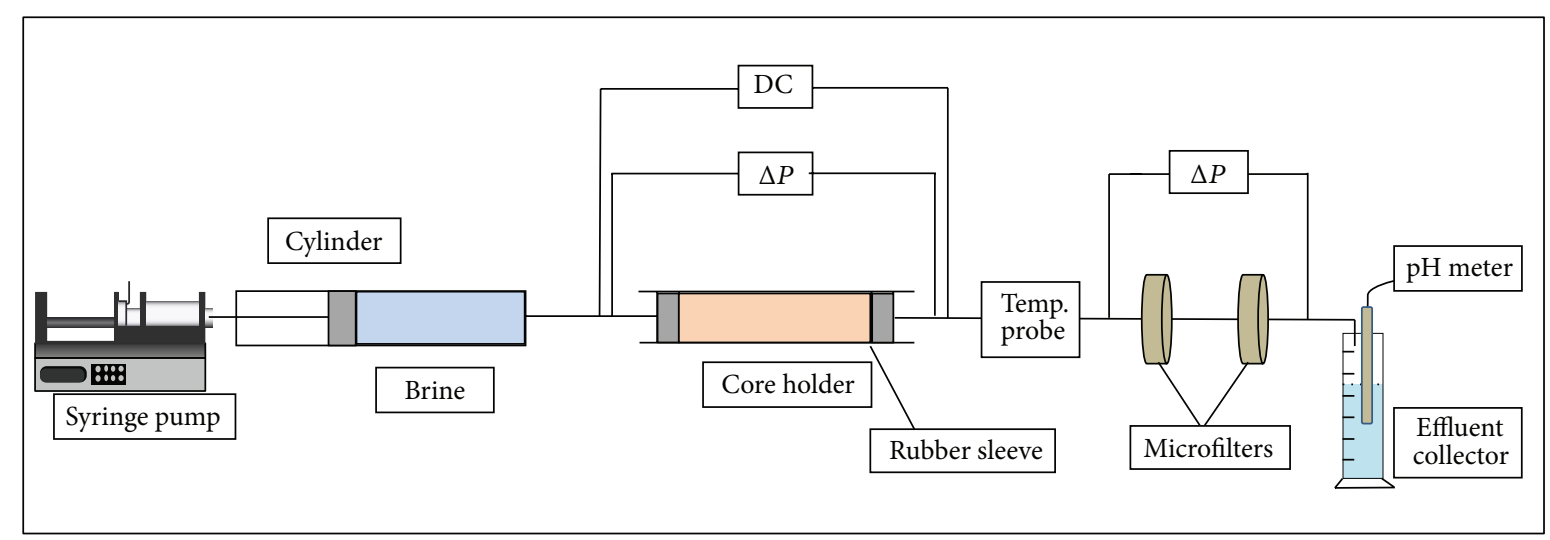

FIGURE 2: Schematics of the flow setup under DC potential.

The objective of this study is to improve injectivity of tight sandstone reservoir taking advantage of the electrokinetic phenomena as described earlier. This is achieved through several coreflood studies in which positive DC potential is applied on the injector side and the negative potential is applied on the reference or production side of low permeable sandstone core plugs, rich in clay minerals. Two sets of experiments are conducted. In the first set, the DC potential is varied and subsequently optimized during the water injection. In the second set, optimum potential is applied and kept constant and the injection rate is varied to determine the hydrodynamic flow effect on clay movement. The liberated clays are characterized through size exclusion microfiltration and mineralogical analysis using ICP-MS technique. The Joule heating phenomena associated with electrokinetics are also studied during the entire injection period.

\section{Methodology}

Low permeability Berea sandstone core plugs of $9^{\prime \prime}$ in length and $1.5^{\prime \prime}$ diameter were used as representative porous media. All plugs were subjected to porosity and $\mathrm{N}_{2}$ permeability measurement. Properties of the selected core plugs are given in Table 1.

An electrically nonconductive coreflooding setup is fabricated. A schematic of the apparatus used for the coreflooding experiments is given in Figure 2. In this set up, we housed the core plug in a rubber sleeve instead of a normal steel core holder and confinement was applied using several adjustable plumber rings. Confinement pressure was adjusted by tightening the rings. A precession positive displacement pump was used to provide the hydrodynamic flow through the core. Two filtration units with $3 \mu \mathrm{m}$ and $1 \mu \mathrm{m}$ filter papers were connected on the production side of the core to collect particles of different sizes produced with the produced brine. Two pressure transducers, sensitive to 2 decimal places of psi, with data logging system were connected as shown in Figure 2. One was installed across the core plug and the other across the filtration units to detect the anticipated pressure rise due to the accumulation of particles and blockage of filter papers. A stable DC power source with current and voltage regulator was used to provide the required potential across the core plug. The whole flooding system was electrically isolated to avoid current leakage and short circuit. The coreflood experiments were conducted as single phase flow with nondamaging $4 \%$ ammonium chloride brine. In addition, arrangements were made to measure the temperature and $\mathrm{pH}$ of the effluent brine. Finally, quantification of produced clay was performed through the use of ICP-MS (inductively coupled plasma-mass spectroscopy), using Perkin Elmer SCIEX DRCe ICP-MS instrument.

Prior to coreflooding, all the core plugs were fully saturated with brine under vacuum followed by saturation under 2000 psi pressure. Absolute brine permeability was measured at a constant flow rate of $0.2 \mathrm{cc} / \mathrm{min}$. Once the differential flow pressure across the core plug is stabilized, a premeditated DC potential is applied and brine flow is continued. Several corefloods were conducted in which the positive potential was applied on the injector side and the negative potential on the reference or production side of the coreflood setup.

The objective of the first set of corefloods is to optimize DC potential gradient, which was varied from $0.5 \mathrm{~V} / \mathrm{cm}$ to $2.0 \mathrm{~V} / \mathrm{cm}$ at an increment of $0.5 \mathrm{~V}$, while brine flow rate was kept constant at $0.2 \mathrm{cc} / \mathrm{min}$. All four corefloods were continued till a steady state was reached. In the second set of corefloods, the main objective is to optimize the flow rate. In this set of four corefloods, the DC potential was kept constant at $1.5 \mathrm{~V} / \mathrm{cm}$, the optimized voltage was obtained from the first set, and the brine injection rates were varied from $0.3 \mathrm{cc} / \mathrm{min}$ up to $0.6 \mathrm{cc} / \mathrm{min}$ at an increment of $0.1 \mathrm{cc} / \mathrm{min}$. A summary of the above two sets of corefloods is presented in Table 2. During the flow studies, effluent temperature was measured to investigate Joule heating effect on the porous media.

\section{Results and Analysis}

Results of the first set of coreflood experiments (Table 2 and Figure 3) show that the application of DC potential had significant impact on clay destabilization and resulting permeability enhancement of the core and is dependent on the applied potential gradient. An apparent increase of core permeability up to $325 \%$ could be seen by application of a 
TABLe 1: Petrophysical properties of core plugs.

\begin{tabular}{|c|c|c|c|c|c|c|}
\hline Core \# & Length $(\mathrm{cm})$ & Diameter $(\mathrm{cm})$ & Porosity by He expansion (\%) & Pore volume (cc) & Absolute permeability $(\mathrm{mD})$ & Exp. \\
\hline 1 & 23.90 & 3.783 & 14.00 & 37.76 & 11.95 & Set 1 \\
\hline 2 & 23.90 & 3.809 & 13.67 & 37.40 & 11.71 & Set 1 \\
\hline 3 & 23.85 & 3.789 & 14.15 & 38.30 & 12.47 & Set 1 \\
\hline 4 & 23.80 & 3.816 & 11.81 & 32.43 & 11.93 & Set 1 \\
\hline 5 & 23.90 & 3.792 & 14.92 & 40.43 & 9.33 & Set 2 \\
\hline 6 & 23.95 & 3.820 & 11.34 & 31.19 & 8.55 & Set 2 \\
\hline 7 & 23.85 & 3.791 & 12.32 & 33.38 & 6.98 & Set 2 \\
\hline 8 & 23.90 & 3.811 & 13.88 & 37.99 & 7.45 & Set 2 \\
\hline
\end{tabular}

TABLE 2: Summary of coreflood experiments and experimental results.

\begin{tabular}{ccccc}
\hline Experiments & Experiment description & WF perm. $(\mathrm{mD})$ & WF + EK perm. $(\mathrm{mD})$ & \% Apparent perm. increase \\
\hline Set 1 & & & & 16.64 \\
Exp. 1 & $0.5 \mathrm{~V} / \mathrm{cm}$ at $0.2 \mathrm{cc} / \mathrm{min}$ & 11.95 & 19.93 & 39.25 \\
Exp. 2 & $1.0 \mathrm{~V} / \mathrm{cm}$ at $0.2 \mathrm{cc} / \mathrm{min}$ & 11.71 & 28.22 & 70.20 \\
Exp. 3 & $1.5 \mathrm{~V} / \mathrm{cm}$ at $0.2 \mathrm{cc} / \mathrm{min}$ & 12.47 & 55.33 & 36.30 \\
Exp. 4 & $2.0 \mathrm{~V} / \mathrm{cm}$ at $0.2 \mathrm{cc} / \mathrm{min}$ & 11.93 & & 21.89 \\
Set 2 & & & 19.94 & 134.62 \\
Exp. 5 & $0.3 \mathrm{cc} / \mathrm{min}$ at $1.5 \mathrm{~V} / \mathrm{cm}$ & 9.33 & 17.59 & 133.22 \\
Exp. 6 & $0.4 \mathrm{cc} / \mathrm{min}$ at $1.5 \mathrm{~V} / \mathrm{cm}$ & 8.55 & 25.30 & 152.01 \\
Exp. 7 & $0.5 \mathrm{cc} / \mathrm{min}$ at $1.5 \mathrm{~V} / \mathrm{cm}$ & 6.98 & & 239.60 \\
Exp. 8 & $0.6 \mathrm{cc} / \mathrm{min}$ at $1.5 \mathrm{~V} / \mathrm{cm}$ & 7.45 & & \\
\hline
\end{tabular}

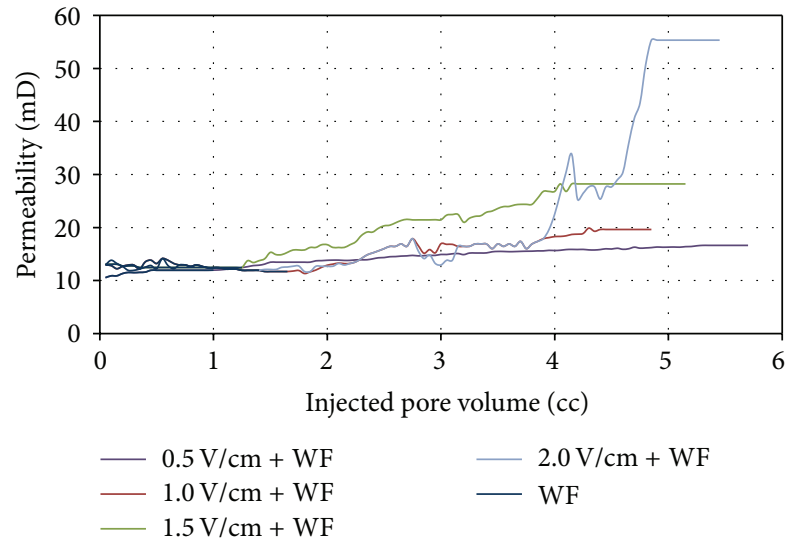

FIGURE 3: Increase of core permeability on application of different DC potential (DC voltage optimization).

potential gradient of $2 \mathrm{~V} / \mathrm{cm}$; however the sudden increase of permeability after approximately $4.5 \mathrm{PV}$ of brine indicates dislodgment of large amount of clays and larger size particles and possibly damaging the matrix itself. It is obvious from Table 3 that larger accumulation of mass on the filters $(>3 \mu \mathrm{m}$ and $>1 \mu \mathrm{m}$ ) was observed in Experiment 4 which indicates large size particle production. Thus, $1.5 \mathrm{~V} / \mathrm{cm}$ potential is considered as optimum, in which slow and progressive increase of permeability is observed (126\%).

In the second set of experiments, the optimized potential $(1.5 \mathrm{~V} / \mathrm{cm})$ was applied and fluid flow rates were varied. It could be seen from the coreflooding results (Table 2 and
TABLE 3: Mass of clay accumulations on size exclusion filters for all experiments.

\begin{tabular}{lcc}
\hline \multicolumn{3}{c}{ Mass of accumulation on filtration units } \\
Experiments & $3 \mu$ paper $(\mathrm{g})$ & $1 \mu$ paper $(\mathrm{g})$ \\
\hline Set 1 & & \\
Exp. 1 & 0.076 & 0.075 \\
Exp. 2 & 0.125 & 0.072 \\
Exp. 3 & 0.144 & 0.072 \\
Exp. 4 & $\mathbf{0 . 5 1 7}$ & $\mathbf{0 . 2 9 8}$ \\
Set 2 & & \\
Exp. 5 & 0.132 & 0.078 \\
Exp. 6 & 0.149 & 0.099 \\
Exp. 7 & 0.163 & 0.104 \\
Exp. 8 & $\mathbf{0 . 4 9 5}$ & $\mathbf{0 . 3 1 5}$ \\
\hline
\end{tabular}

Figure 4) that upon the application of a fixed voltage, permeability increased from its base value as the hydrodynamic flow rate was increased. An apparent increase of core permeability up to $240 \%$ could be observed by application of $0.6 \mathrm{cc} / \mathrm{min}$ flow rate. However, the sudden increase of permeability after approximately 4.5 PV of brine implies dislodgement of larger size particles which was observed in the size exclusion filters (Table 3). Therefore, $0.5 \mathrm{cc} / \mathrm{min}$ injection rate is considered as optimum in terms of slow and gradual increase of permeability (152\%).

A comparison of the results from the two sets of experiments shows that the effect of DC potential is more 


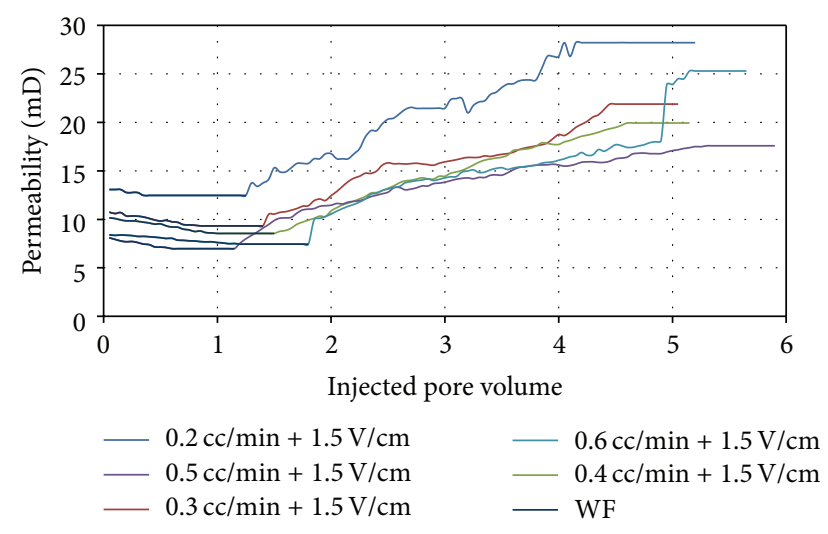

Figure 4: Permeability changes results from set 2 experiments (injection rate optimization).

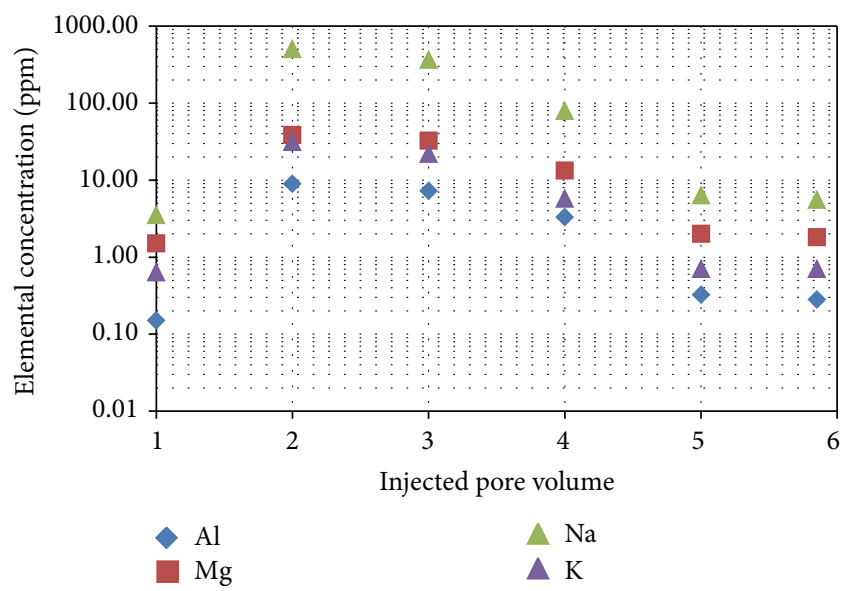

FIGURE 5: ICP-MS analysis results $(1.5 \mathrm{~V} / \mathrm{cm}$ and $0.5 \mathrm{cc} / \mathrm{min})$.

pronounced than the effect of hydrodynamic potential. ICPMS analysis results of the effluents collected at each PV of brine injection (after application of DC potential) show metal elements corresponding to clay minerals in abundant quantity (Figure 5), whereas the quantity of clay trapped in the filter papers is much less. This indirectly indicates that the clays produced with effluents are smaller than 1 micron in size and flow easily through the pore channels (Table 3 ). It is also evident from this plot that the concentration of the relevant clay elements is seen to be reduced drastically after 5 pore volumes of water injection when the stabilized conditions are achieved and no further clay production is noticed.

Temperature measurements during the flow studies showed an increasing trend with the injection time and direct proportionality with the applied voltages (Figure 6). Nevertheless, the behavior of temperature changes is similar among set 2 experiments upon which the same potential gradient was applied for all the flow experiments (Figure 7). This is due to the fact that Joule heating is proportional to the applied voltage gradient.

$\mathrm{pH}$ measurements of the effluent samples (Figure 8) show initial increase of $\mathrm{pH}$ followed by a stabilization period till the end of the flow, indicating alkaline environment on

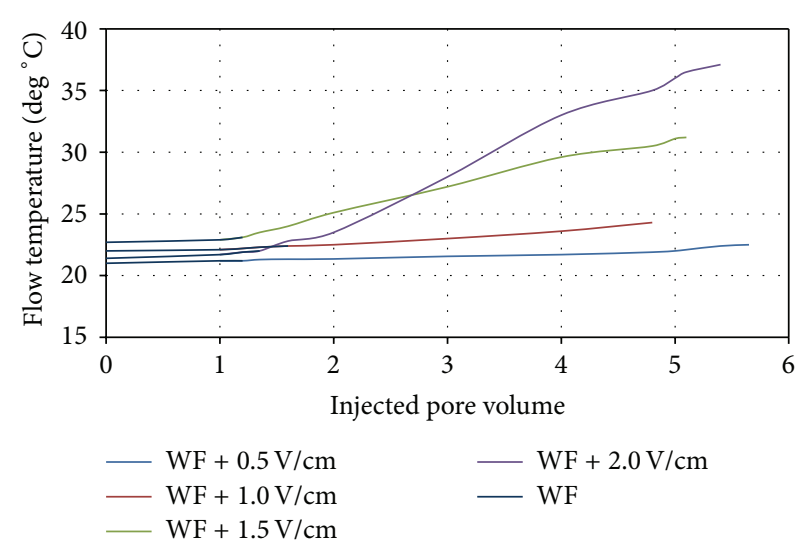

FIGURE 6: Temperature change upon application of different DC potential.

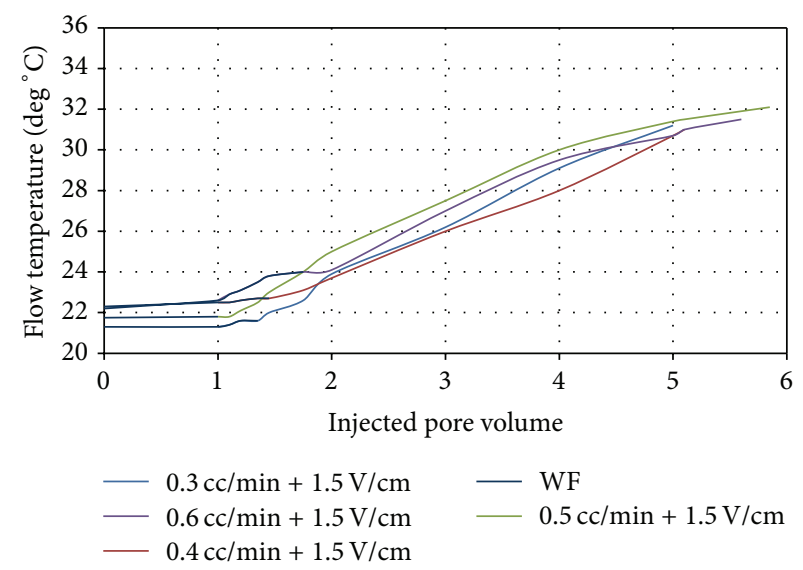

Figure 7: Temperature change at different flow rates at $1.5 \mathrm{~V} / \mathrm{cm}$.

application of DC potential. As mentioned earlier, differential pressure across the filters is measured on continuous basis to monitor plugging of filter papers; this is represented by Figure 9. From this figure, it is evident that there is no increase in $\Delta P$ during the brine flow, indicating no clay or fine production. However, after commencing electrokinetic assisted flow, the $\Delta P$ across the filters increased rapidly at the initial period, slowed down after about $0.5 \mathrm{PV}$ of brine injection, and finally stabilized after $5 \mathrm{PV}$ of injection.

\section{Discussion}

Overall observation from the above results shows that water injection efficiency can be enhanced by application of DC potential. Table 2 illustrates this phenomenon, depicting a linear correlation between applied voltage and permeability increase or core stimulation. This phenomenon can be explained only by the fact that clay disintegration and migration in submicron sizes take place under the influence of various electrokinetic mechanisms as discussed earlier. It is known that the structure of clay mineral is capable of changing the sign of their surface potential with a change in $\mathrm{pH}$ of the medium. In an acidic medium, the surface of 


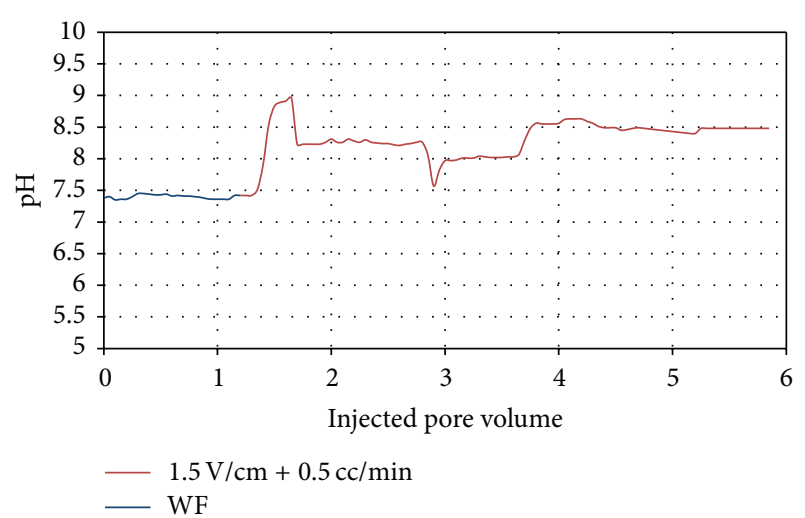

FIGURE 8: $\mathrm{pH}$ measurements at optimized conditions $(1.5 \mathrm{~V} / \mathrm{cm}$ and $0.5 \mathrm{cc} / \mathrm{min})$.

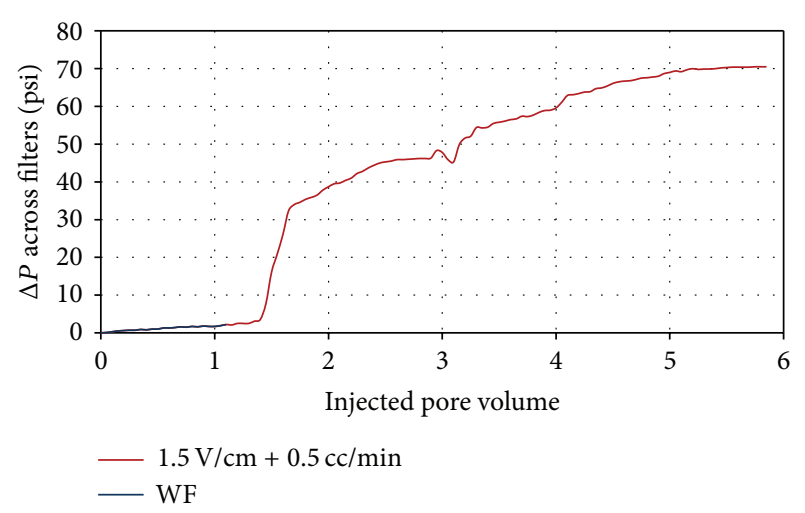

FIgURE 9: Differential pressure change across filtration units $(1.5 \mathrm{~V} / \mathrm{cm}$ and $0.5 \mathrm{cc} / \mathrm{min})$.

clay particles is seen to be heteropotential, the basal surfaces gain a net negative potential, and the edges gain a net positive potential [20], whereas in the alkaline medium, the basal surface of the mineral and their edges bear the same negative potential. This is due to the fact that $\mathrm{Al}$ and Fe hydroxides (which make part of the crystalline structure of the clay minerals) show amphoteric properties and depending on the $\mathrm{pH}$ of the medium may have excess of negative or positive charges [21].

In the single phase flow experiment with $\mathrm{NH}_{4} \mathrm{Cl}$ brine, the net flow of ions towards the producer cathode is $\mathrm{NH}_{4}{ }^{+}$ and $\mathrm{H}^{+}$. Being a bulky ion, $\mathrm{NH}_{4}{ }^{+}$will find it difficult to penetrate between two basal plates of clay matrix; however the tiny $\mathrm{H}^{+}$ions would easily penetrate and concentrate on the basal surface. Thus, the production and flow of $\mathrm{H}^{+}$ions from the anode (injector side) towards cathode (producer side) may have twofold impacts on the clay structures; (1) it would increase the electrostatic repulsion between layers due to excess of positive charges between the layers and increase the distance between them; (2) it would introduce local changes in the $\mathrm{pH}$ and make the clay particles heteropotential, which in turn change the clay structures from a compact layered structure to colloidal "house of cards" structure as shown in Figure 1. With the help of the hydrodynamic and electroosmotic potential, the submicron size colloidal particles would easily flow through the micron size pore channels, increasing pore throat sizes, and enhance core permeability. This explanation is supported by the high content of metals ( $\mathrm{Na}, \mathrm{Al}, \mathrm{Mg}$, and $\mathrm{K}$ ) in the produced brine (ICP-MS analysis, Figure 5), which are naturally present in the clays. The impact of DC potential on clay disintegration and migration is also supported by the fact that filter paper clogging and resulting pressure rise were observed only after the application of DC potential and not during normal brine flooding.

\section{Conclusion}

The study aimed to investigate an alternate method to improve water injectivity in tight sandstone reservoir. The findings of this study can be summarized as follows.

(i) Application of DC potential enhances the permeability of tight clay rich sandstone rocks which further boosts water injection efficiency.

(ii) Clay disintegration and mobilization are believed to be one of the main contributing mechanisms to permeability enhancement by electrokinetics in sandstone rocks. However, the effect of the application of DC potential on the mineralogy of clays yet needs to be thoroughly investigated.

(iii) Permeability enhancement increases as both applied potential gradient and hydrodynamic injection rate increase.

(iv) The application of carefully studied injection rate and potential difference may facilitate the injectivity of water, based on the rock characteristics.

(v) Flow temperature measurements showed an increasing trend with the injection time and direct proportionality with applied voltages.

(vi) $\mathrm{pH}$ of the effluent is seen to increase when the flow was subjected under DC potential, which may have impact on clay disintegration.

\section{Nomenclature}

A: Cross-sectional area of flow, $\mathrm{m}^{2}$

E: Applied electrical potential, $\mathrm{V}$

$K$ : Absolute permeability, $\mathrm{m}^{2}$ or $\mathrm{D}$

$k_{e}$ : Intrinsic electroosmotic permeability, $\mathrm{Pa} \cdot \mathrm{m}^{2} / \mathrm{V}$

$L: \quad$ Length of core, $\mathrm{m}$

$\Delta p$ : Pressure difference, $\mathrm{Pa}$

$M$ : Dynamic viscosity, Pa.s.

\section{Conflict of Interests}

The authors declare that there is no conflict of interests regarding the publication of this paper.

\section{Acknowledgment}

The authors are grateful to the Petroleum Engineering Department at The Petroleum Institute, Abu Dhabi, for 
providing the laboratory facilities to conduct the laboratory experiments.

\section{References}

[1] T. Ahmed, Reservoir Engineering Handbook, Gulf Professional Publishing, 2nd edition, 2001.

[2] P. Willhite, Waterflooding, SPE Textbook Series, vol. 3, Society of Petroleum Engineers, 1986.

[3] S. Pang and M. Sharma, "A model for predicting injectivity decline in water-injection wells," SPE Formation Evaluation, vol. 3, no. 12, pp. 194-201, 1997.

[4] A. Tchistiakov, "Physio-chemical aspects of clay migration and injectivity decrease of geothermal clastic reservoirs," in Proceedings of the World Geothermal Congress (WGC '00), 2000.

[5] M. A. Miniawi, W. A. F. Ahmed, Y. A. Ahmed, and A. R. Soufi, "In situ gelled acid as a diverting system in water injection well," in Proceedings of the SPE/IADC Middle East Drilling Technology Conference and Exhibition, pp. 333-337, October 2007.

[6] X. Yi, "Water injectivity decline caused by sand mobilization: simulation and prediction," in Proceedings of the SPE Permian Basin Oil and Gas Recovery Conference, Paper SPE 70032, pp. 202-209, Midland, Tex, USA, May 2001.

[7] B. Scarth and C. Vozniak, "Production problems associated with overstressed hydraulic fracture treatments," Paper SPE 89-4079, Presented at Annual Technical Meeting, Banff, Canada, 1989.

[8] X. Wang, H. Zou, X. Cheng et al., "A new approach for matrix acidizing of water injectors in low-permeability Sandstone fields," Paper SPE 73780, Presented at International Symposium and Exhibition on Formation Damage Control, Lafayette, La, USA, 2012.

[9] H. A. Al-Anazi, H. A. Nasr-El-Din, M. K. Hashem, and J. A. Hopkins, "Matrix acidizing of water injectors in a sandstone field in Saudi Arabia: a case study," in Proceedings of the SPE/AAPG Western Regional Meetings, Paper SPE 62825, pp. 585-594, Long Beach, Calif, USA, June 2000.

[10] S. A. Amba, G. V. Chilingar, and C. M. Beeson, "Application of electrokinetics phenomena in civil and petroleum engineering," The New York Academy of Sciences, vol. 118, no. 14, pp. 585-602, 1965.

[11] S. A. Amba, G. V. Chilingar, and C. M. Beeson, "Use of direct electrical current for increasing the flow rate of reservoir fluids during petroleum recovery," Journal of Canadian Petroleum Technology, vol. 3, no. 1, pp. 8-14, 1964.

[12] G. Chilingar and C. Beeson, "Use of direct electrical current for increasing the flow rate of oil and water in a porous medium," Journal of Canadian Petroleum Technology, vol. 4, no. 1, pp. 81$88,1965$.

[13] M. R. Haroun, G. V. Chilingar, S. Pamukcu, J. K. Wittle, H. A. Belhaj, and M. N. A. Bloushi, "Optimizing electroosmotic flow potential for electrically enhanced oil recovery (EEOR) in carbonate rock formations of Abu Dhabi based on rock properties and composition," in Proceedings of the International Petroleum Technology Conference (IPTC '09), Paper SPE 13812, pp. 2645-2659, Doha, Qatar, December 2009.

[14] J. K. Wittle, D. G. Hill, and G. V. Chilingar, "Direct current electrical enhanced oil recovery in heavy-oil reservoirs to improve recovery, reduce water cut, and reduce $\mathrm{H} 2 \mathrm{~S}$ production while increasing API gravity," in Proceedings of the SPE Western Regional and Pacific Section AAPG Joint Meeting (SPE '08), pp. 405-423, Bakersfield, Calif, USA, April 2008.
[15] G. Chilingar, A. El-Nassir, and R. Steven, "Effect of direct electrical current on permeability of sandstone core," Journal of Petroleum Technology, vol. 22, no. 7, pp. 830-836, 1970, Paper SPE 2332.

[16] J. E. Killough and J. A. Gonzalez, "A fully-implicit model for electrically enhanced oil recovery," in Proceedings of the 61st Annual Technical Conference and Exhibition, New Orleans, La, USA, October 1986.

[17] S. Pamukcu, "Electrochemical transport and transformations," in Chapter 2 in Electrochemical Remediation Technologies for Polluted Soils, Sediments and Groundwater, Reddy and Camaselle, Eds., pp. 29-65, John Wiley \& Sons, New York, NY, USA, 2009.

[18] D. H. Gray and J. K. Mitchell, "Fundamental aspects of electroosmosis in soils," Journal of the Soil Mechanics and Foundations Division, vol. 93, no. 6, pp. 209-236, 1967.

[19] B. Ghosh, E. W. Al Shalabi, and M. Haroun, "The effect of DC electrical potential on enhancing sandstone reservoir permeability and oil recovery," Petroleum Science and Technology, vol. 30, no. 20, pp. 2148-2159, 2012.

[20] V. Sokolov, “Models of clay soil microstructures," Inzhenernaya Geologiya, no. 6, pp. 32-42, 1991.

[21] V. I. Osipov, V. N. Sokolov, and V. V. Eremeev, Clay Seals of Oil and Gas Deposits, Balkema, Rotterdam, The Netherlands, 2004. 

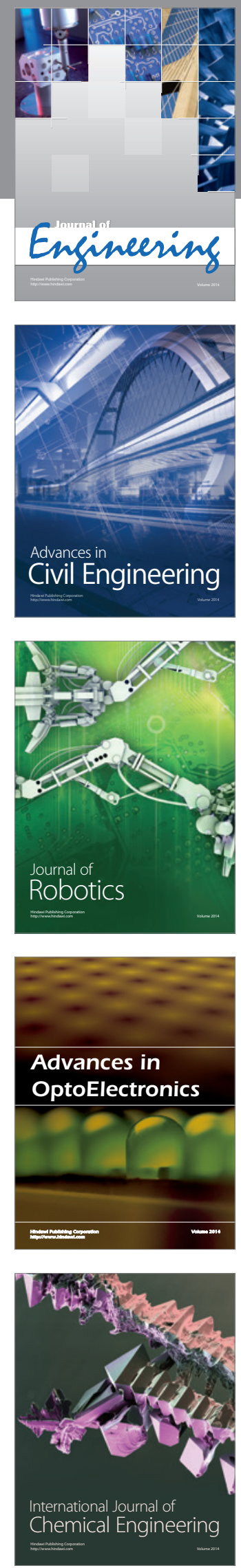

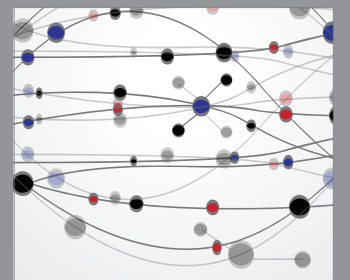

The Scientific World Journal
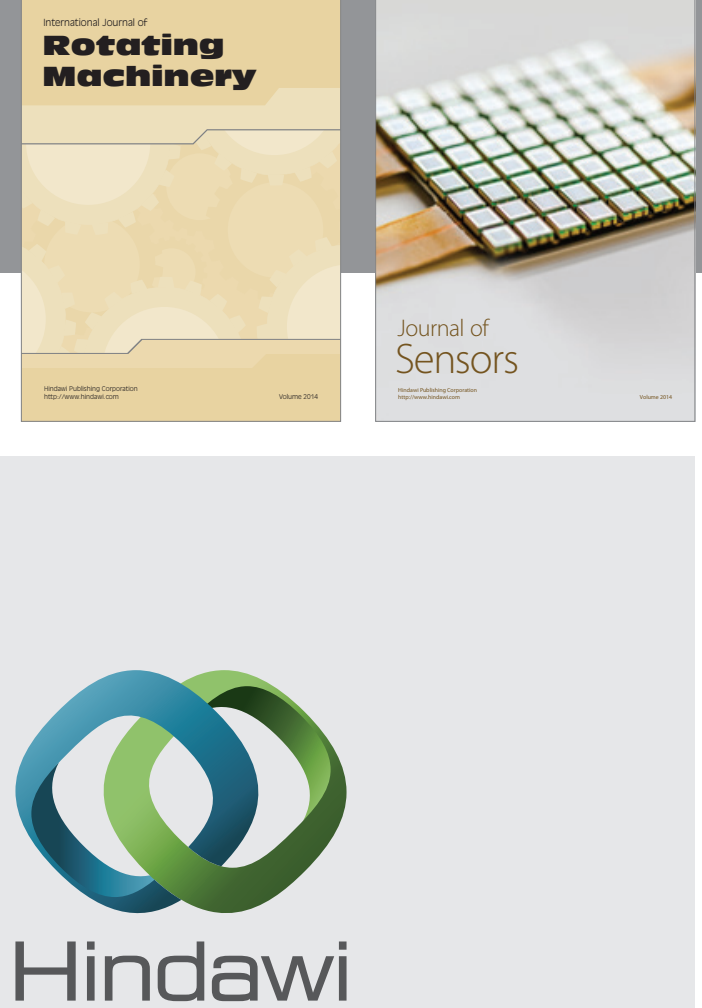

Submit your manuscripts at http://www.hindawi.com
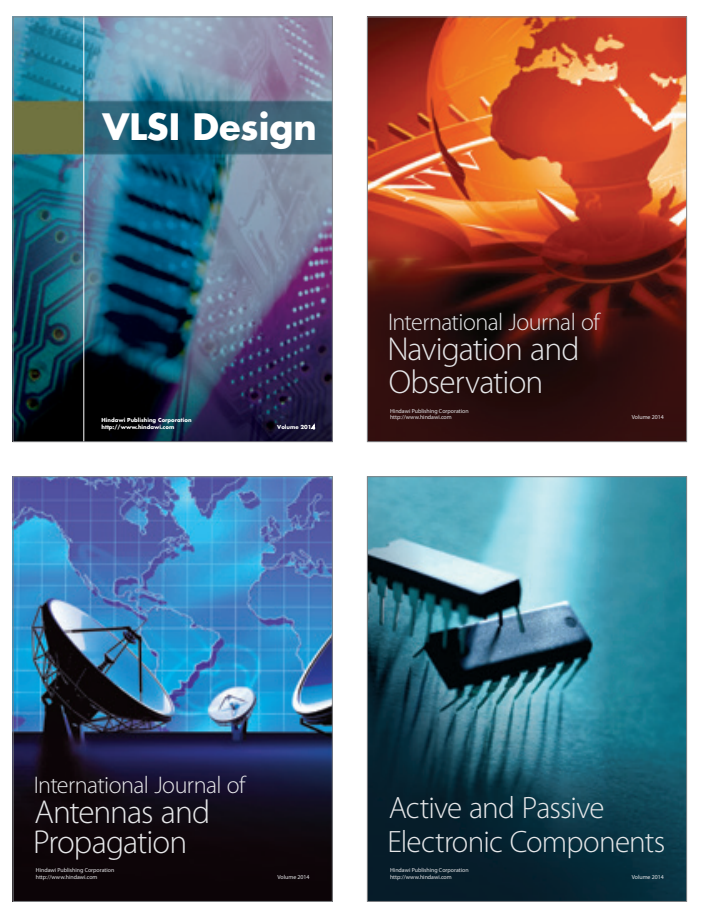
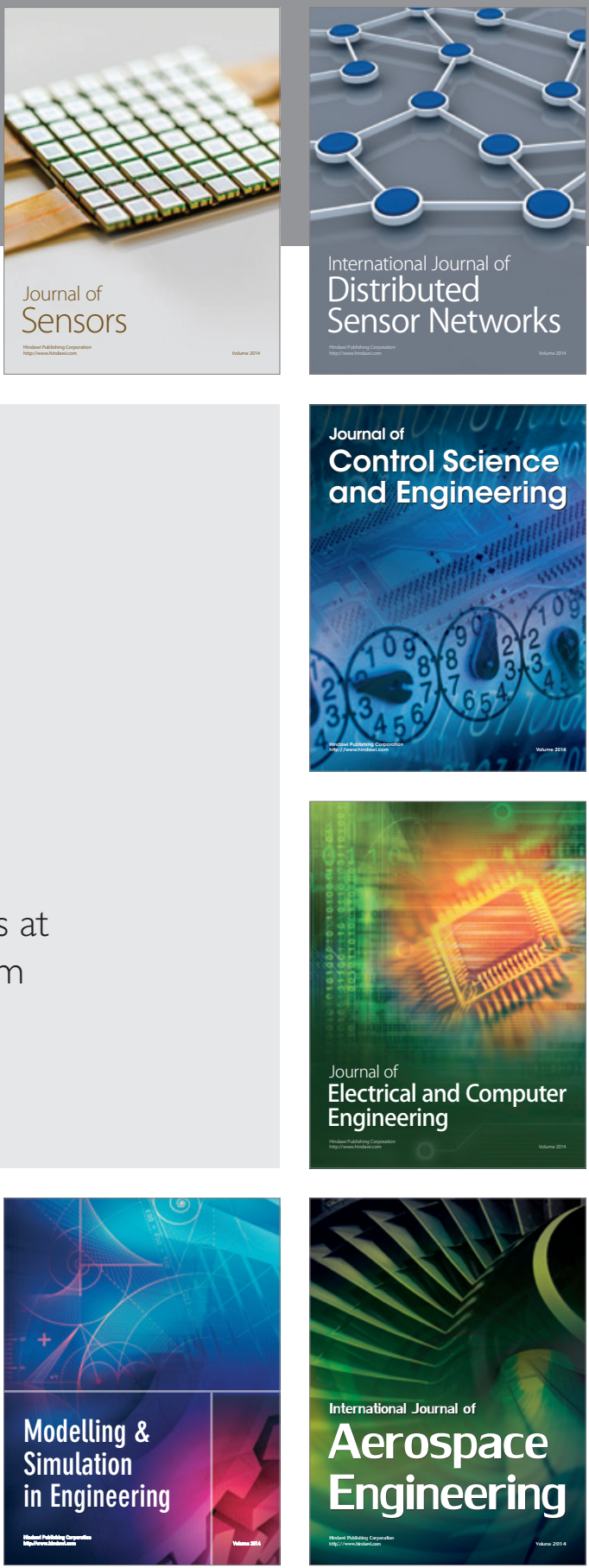

Journal of

Control Science

and Engineering
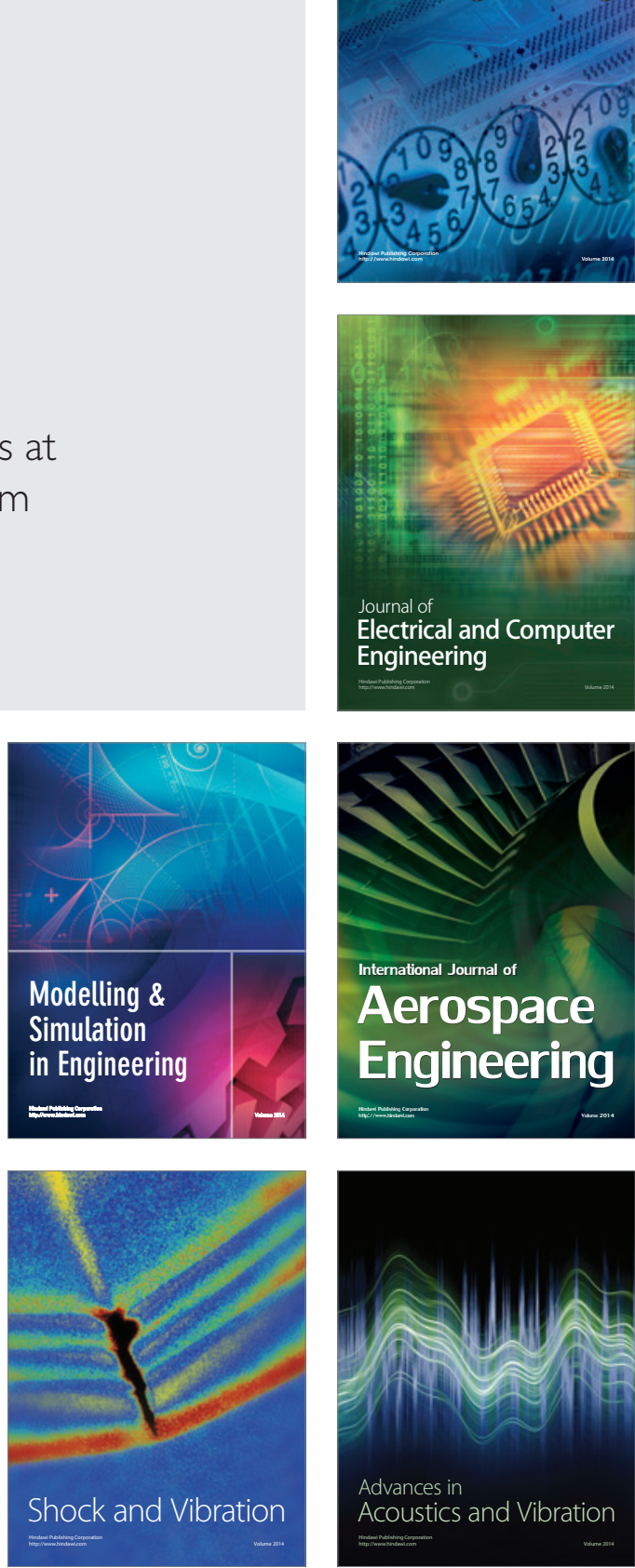